CARDIOVASCULAR MEDICINE

\title{
Relation of arterial stiffness to diastolic dysfunction in hypertensive heart disease
}

\author{
P M Mottram, B A Haluska, R Leano, S Carlier, C Case, T H Marwick
}

Heart 2005;91:1551-1556. doi: 10.1136/hrt.2004.046805

See end of article for
authors' affiliations
......................
Correspondence to:
Professor Thomas
H Marwick, University of
Queensland Department of
Medicine, Princess
Alexandra Hospital,
lpswich Road, Brisbane,
Q4102, Australia;
tmarwick@soms.uq.edu.au
Accepted
14 December 2004

\begin{abstract}
Objectives: To examine the relation of arterial compliance to diastolic dysfunction in hypertensive patients with suspected diastolic heart failure (HF).

Patients: 70 medically treated hypertensive patients with exertional dyspnoea (40 women, mean (SD) age 58 (8) years) and 15 normotensive controls.

Main outcome measures: Mitral annular early diastolic velocity with tissue Doppler imaging and flow propagation velocity were used as linear measures of diastolic function. Arterial compliance was determined by the pulse pressure method.

Results: According to conventional Doppler echocardiography of transmitral and pulmonary venous flow, diastolic function was classified as normal in 33 patients and abnormal in 37 patients. Of those with diastolic dysfunction, 28 had mild (impaired relaxation) and nine had advanced (pseudonormal filling) dysfunction. Arterial compliance was highest in controls (mean (SD) $1.32(0.58) \mathrm{ml} / \mathrm{mm} \mathrm{Hg}$ ) and became progressively lower in patients with hypertension and normal function $(1.04(0.37) \mathrm{ml} / \mathrm{mm} \mathrm{Hg})$, impaired relaxation $(0.89(0.42) \mathrm{ml} / \mathrm{mm} \mathrm{Hg})$, and pseudonormal filling $(0.80(0.45) \mathrm{ml} / \mathrm{mm} \mathrm{Hg}, p=0.011)$. In patients with diastolic dysfunction, arterial compliance was inversely related to age $(p=0.02)$, blood pressure $(p<0.001)$, and estimated filling pressures $(p<0.01)$ and directly related to diastolic function $(p<0.01)$. After adjustment for age, sex, body size, blood pressure, and ventricular hypertrophy, arterial compliance was independently predictive of diastolic dysfunction.

Conclusions: In hypertensive patients with exertional dyspnoea, progressively abnormal diastolic function is associated with reduced arterial compliance. Arterial compliance is an independent predictor of diastolic dysfunction in patients with hypertensive heart disease and should be considered a potential target for intervention in diastolic HF.
\end{abstract}

$\mathrm{T}$ he association between arterial dysfunction and left ventricular (LV) systolic dysfunction is well characterised, but many patients with hypertension have exercise limitation but normal systolic function, raising the question of whether this could be due to diastolic dysfunction. Possibly, this reflects a common aetiology of arterial and ventricular disease: risk factors for diastolic heart failure (HF) (hypertension, increasing age, and atherosclerosis) are also associated with a reduction in arterial compliance. However, abnormal arterial compliance may potentially contribute to the development of LV diastolic dysfunction through increased pulse pressure and LV afterload, which in turn promote LV hypertrophy and subendocardial ischaemia. ${ }^{1}$ Indeed, reduced aortic distensibility has recently been reported in patients with diastolic HF and correlates with exercise limitation in these patients. ${ }^{2}$ However, the relation of total arterial compliance to diastolic dysfunction in hypertensive heart disease remains unclear. The objectives of the study were to examine the relation of arterial compliance to the stage of diastolic dysfunction in hypertensive patients with suspected diastolic HF, to determine whether arterial compliance is independently associated with abnormal myocardial function in these patients, and to assess the interaction of sex with arterial compliance and diastolic dysfunction in this population.

\section{METHODS}

\section{Patients}

We prospectively studied 85 ambulatory patients from the community: 70 patients (40 women, mean age 58 (8) years) with medically treated hypertension and exertional dyspnoea (New York Heart Association class II) and 15 normotensive controls. All patients were clinically stable, with none requiring admission to hospital. Participants were clinically assessed by a cardiologist followed by screening ECG and echocardiography. Only patients with normal resting LV systolic function (ejection fraction $>50 \%$ and LV end diastolic internal dimension index $<3.2 \mathrm{~cm} / \mathrm{m}^{2}$ ) and without significant (more than mild) valve dysfunction were enrolled. Patients also underwent exercise ECG or exercise echocardiography under the Bruce protocol, with evidence of ischaemia defined by ischaemic ECG changes or a new wall motion abnormality during stress. Patients with evidence of ischaemic heart disease were not eligible for the study. Such evidence included a history suggestive of angina (as taken by a cardiologist), history of unstable angina or myocardial infarction, Q waves on ECG, regional wall motion abnormality on echocardiography, or evidence of ischaemia during stress testing. B-type natriuretic peptide (BNP) was measured in a subgroup of the hypertensive patients $(n=64)$ by the Triage assay point of care system (Biosite Diagnostics, San Diego, California, USA) as previously described. ${ }^{3}$ Normal or abnormal diastolic function was identified on the basis of Doppler echocardiography (see below).

\section{Echocardiography}

A detailed two dimensional Doppler echocardiogram (Vivid Five, GE Vingmed, Milwaukee, Wisconsin, USA) was recorded for all patients. M mode measurements of end

Abbreviations: A, peak late diastolic transmitral velocity; BNP, B-type natriuretic peptide; $E$, peak early diastolic transmitral velocity; Ea, peak early diastolic mitral annular velocity; HF, heart failure; LV, left ventricular; $\mathrm{Vp}$, flow propagation velocity 


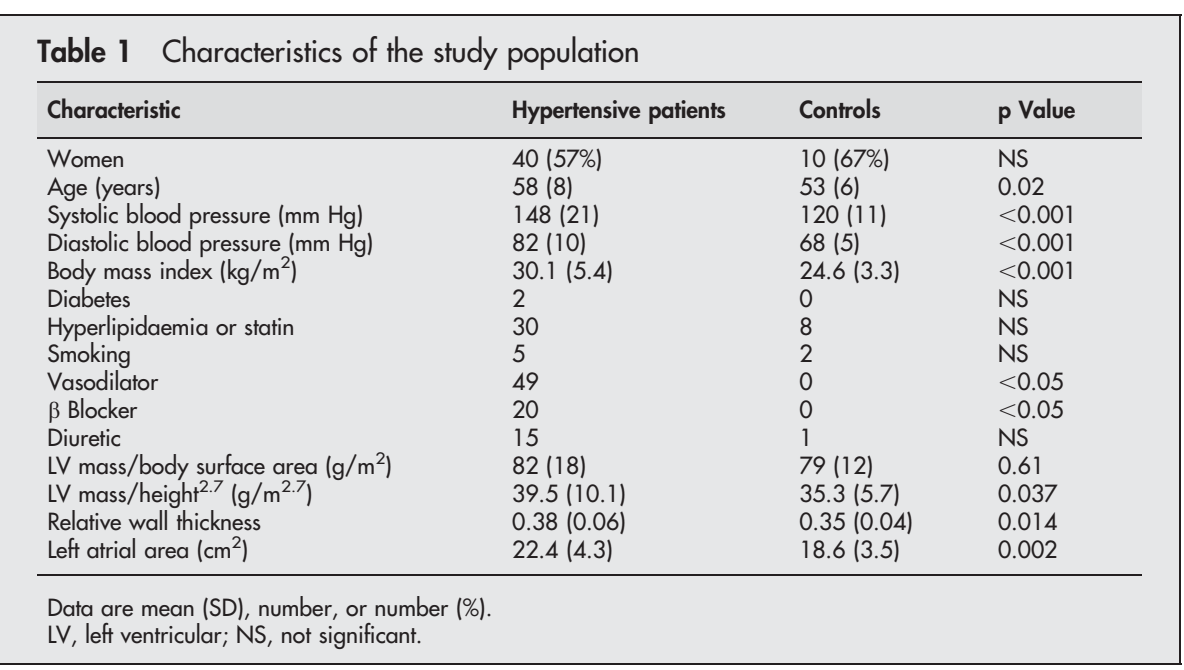

diastolic LV wall thickness and cavity diameter were used for calculation of LV mass ${ }^{4}$ and indexed to body surface area or height. Relative wall thickness was defined by the sum of the septal and posterior wall thicknesses divided by the end diastolic dimension. Left atrial area was traced in an apical four chamber view. Comprehensive assessment of LV diastolic function included transmitral and pulmonary vein pulsed wave Doppler from an apical four chamber view. Transmitral early (E) and late (A) diastolic velocities, E wave deceleration time, and A wave duration were successfully recorded for all patients. Systolic and diastolic velocities were measured in the right upper pulmonary vein, as were the velocity and duration of the atrial reversal wave. The A reversal peak velocity was obtained for all patients; the pulmonary venous systolic and diastolic velocities and the A duration were obtainable for 83 of the 85 participants. Diastolic function was classified as normal, impaired relaxation, pseudonormal, or restrictive according to standard diagnostic criteria. ${ }^{5}$ Normal diastolic function was defined by E:A of 0.75-1.5 and a deceleration time of 160-250 ms. Impaired LV relaxation was defined by the combination of $\mathrm{E}: \mathrm{A}<1$ and $\mathrm{E}$ wave deceleration time $>250 \mathrm{~ms}^{.5-7}$ Pulmonary vein velocities were used to differentiate normal from pseudonormal filling in patients who had a normal transmitral Doppler profile. A pseudonormal LV filling pattern was defined by E:A > 1 that reversed with Valsalva manoeuvre, by pulmonary venous A duration - transmitral A duration $>30 \mathrm{~ms}$, or by pulmonary venous A reversal velocity $>35 \mathrm{~cm} / \mathrm{s}^{.568}$ No patients had a restrictive filling pattern.

Tissue Doppler imaging parameters and LV flow propagation velocity $(\mathrm{Vp})$ were also obtained as more linear (less load dependent) measures of diastolic function ${ }^{9}{ }^{10}$ for correlation with arterial compliance in a multivariate model. Systolic and early (Ea) and late diastolic velocities were measured at the septal and lateral mitral annulus with pulsed wave tissue Doppler imaging in the apical four chamber view. The transducer was positioned to align the ultrasound beam with longitudinal LV motion. A sample volume of $2-5 \mathrm{~mm}$ was used with the frame rate exceeding 100 frames/s. Gains were reduced to minimise spectral broadening and digital images were analysed off line with commercial software (Echopac, GE Vingmed). The intraobserver variation was a mean (SD) of $0.4(0.4) \mathrm{cm} / \mathrm{s}$ with a coefficient of variation of $8 \%$. The ratio of $\mathrm{E}$ to lateral $\mathrm{Ea}$ was used to estimate LV filling pressures. ${ }^{11}{ }^{12} \mathrm{Vp}$ was measured with colour $\mathrm{M}$ mode as the slope of the first aliasing velocity of the early diastolic signal from the plane of the mitral valve into the LV cavity. ${ }^{13}$ Parameters were measured off line and averaged from three consecutive cardiac cycles. Tissue Doppler imaging parameters and Vp were successfully obtained for all participants.

\section{Arterial compliance}

Total arterial compliance was measured by the pulse pressure method after 10 minutes of supine rest. This method derives total arterial compliance from measured aortic flow and peripheral resistance. ${ }^{14}$ Aortic flow was derived from the product of LV outflow tract area and the velocity time integral obtained at echocardiography. Mean and diastolic brachial pressures (Dinamap Plus, Critikon, Tampa, Florida, USA) were used to calibrate the pressure signal derived by applanation tonometry (SPT-301 Mikro-Tip, Millar Instruments, Houston, Texas, USA) at the radial artery, which, being closer to the surface than the brachial artery, makes tonometry more feasible. A transfer function, validated in an angiographic population ${ }^{15}$ and applied previously in a group similar to the current patient population, ${ }^{16}$ was then used to determine mean aortic pressure. Simultaneous gated signals from tonometry, pulsed wave Doppler, and the ECG were acquired simultaneously by an analogue to digital board (WaveBook 512, IOTech Inc, Cleveland, Ohio, USA) attached to a personal computer. Data were analysed by a custom program written in MATLAB 4.2c (The MathWorks, Natick, Massachusetts, USA). Between 20 and 30 cardiac cycles were acquired; depending on data quality, 4-15 specific cardiac cycles were chosen from the raw data set and averaged for analysis. The analysis program determined mean values for pressure and flow and calculated mean aortic pressure by means of the transfer function. Total arterial compliance was then derived from pressure and flow data. The intraobserver coefficient of variation was $1.7 \%$.

\section{Statistical analysis}

Continuous variables are presented as mean (SD). Group differences in continuous variables were assessed with unpaired Student's $t$ test or one way analysis of variance as appropriate. Subgroup differences were analysed post hoc by the Bonferroni correction for multiple comparisons. Correlations between continuous and categorical variables were assessed with Pearson or Spearman methods as appropriate. The independent predictors of arterial compliance and diastolic dysfunction were examined with backwards stepwise linear regression including univariate correlates after adjustment for co-linearity. In addition, multivariate models of diastolic function that included age and LV mass were also assessed. The significance of any interaction between arterial compliance and sex on diastolic function was assessed with an interaction term in a general 


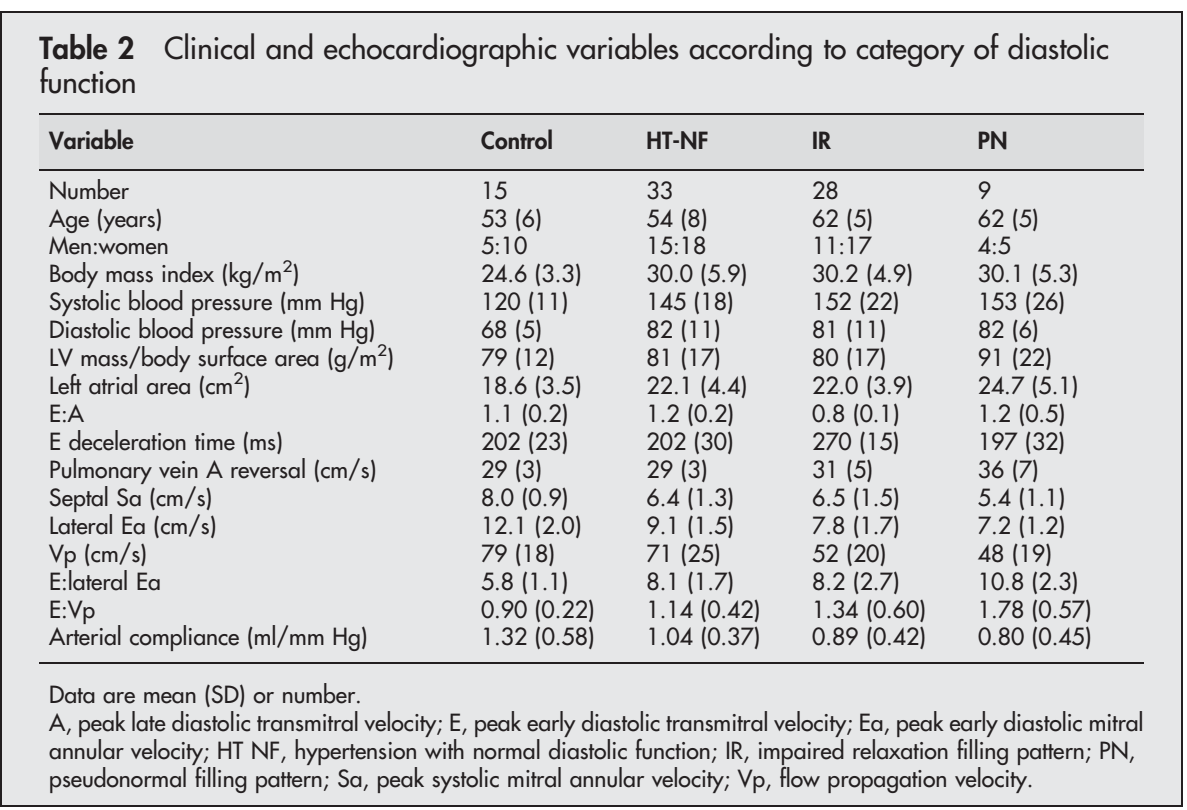

linear model. BNP was not included in multivariate analysis, as it was measured in only a subgroup of patients. Data were analysed with standard statistical software (SPSS version 9; SPSS Inc, Chicago, Illinois, USA). A probability value of $\mathrm{p}<0.05$ was considered significant.

\section{RESULTS}

Table 1 presents the characteristics of the study participants. Women slightly outnumbered men. Hypertensive patients were overweight compared with controls. In the group as a whole, diastolic blood pressure was better controlled than systolic pressure. The majority of patients were taking vasodilator medication, which consisted of a calcium channel blocker for 42 patients, an angiotensin converting enzyme inhibitor for six patients, and prazosin for two patients. Diuretic medication was either thiazide or loop diuretics, with no patients taking aldosterone antagonists. Relative wall thickness and LV mass indexed to height were increased in hypertensive patients, as was left atrial area. As a result of the increased body mass index in hypertensive patients, LV mass indexed to body surface area in this group was similar to that of controls. Among the 70 patients with hypertension, conventional Doppler echocardiography showed normal diastolic function in 33 patients and abnormal diastolic function in 37 patients ( 22 women). The majority of those with abnormal diastolic function had an impaired relaxation pattern $(\mathrm{n}=28)$, whereas a pseudonormal LV filling profile

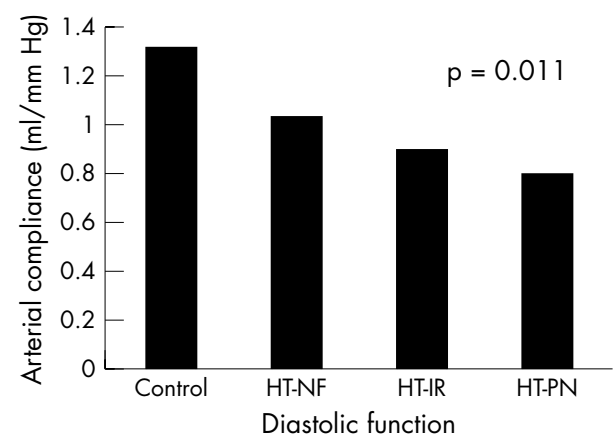

Figure 1 Arterial compliance by classification of diastolic function. HT, hypertension; IR, impaired left ventricular (LV) relaxation; NF, normal diastolic function; PN, pseudonormal LV filling. was documented in nine patients. Table 2 presents the clinical and echocardiographic variables according to category of diastolic function.

Arterial compliance by category of diastolic function Figure 1 compares arterial compliance between controls and hypertensive patients according to category of diastolic function. Arterial compliance was highest in controls and was progressively reduced in hypertensive patients with increasingly abnormal diastolic function $(p=0.011$ by analysis of variance), being lowest in patients with advanced diastolic dysfunction. The Bonferroni correction detected significant differences in arterial compliance between controls and the impaired relaxation group $(p=0.019)$, and between controls and the pseudonormal group $(\mathrm{p}=0.034)$.

Table 3 Analysis of arterial compliance in all hypertensive patients

\begin{tabular}{lll}
\hline Univariate correlate & $\boldsymbol{r}$ & $\mathbf{p ~ V a l u e ~}$ \\
\hline Age & -0.34 & 0.004 \\
Sex & 0.36 & 0.003 \\
Height & 0.30 & 0.012 \\
Weight & 0.31 & 0.009 \\
Body surface area & 0.34 & 0.004 \\
Systolic blood pressure & -0.69 & $<0.001$ \\
Diastolic blood pressure & -0.20 & 0.116 \\
Pulse pressure & -0.75 & $<0.001$ \\
Pulmonary vein A reversal & -0.26 & 0.029 \\
Septal Sa & 0.36 & 0.002 \\
Septal Ea & 0.24 & 0.045 \\
Lateral Ea & 0.35 & 0.003 \\
Vp & 0.30 & 0.011 \\
E:Ea & -0.24 & 0.045 \\
E:Vp & -0.26 & 0.032 \\
BNP & -0.30 & 0.017 \\
\hline Multivariate predictor & $\boldsymbol{\beta}$ & $\mathbf{p ~ V a l u e}$ \\
\hline Body surface area & & 0.011 \\
Pulse pressure & 0.20 & $<0.001$ \\
Septal Sa & -0.68 & 0.065 \\
Vp & 0.14 & 0.042 \\
\hline BNP, B-type natriuretic peptide. & 0.16 & \\
$R^{2}=0.67$. & & \\
\hline
\end{tabular}



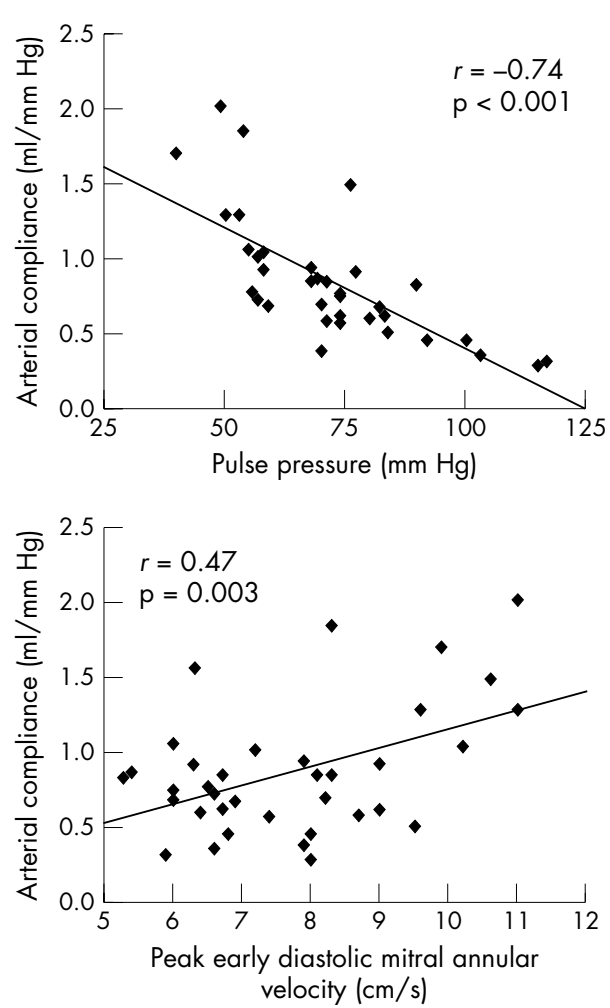

Figure 2 Relation of arterial compliance to (top) pulse pressure and (bottom) lateral early diastolic mitral annular velocity in patients with diastolic dysfunction.

\section{Relations of arterial compliance in hypertensive patients}

Table 3 presents the univariate correlates of arterial compliance in the hypertensive population. As expected, arterial compliance was inversely associated with age and blood pressure, pulmonary venous A reversal velocity (a marker of LV compliance), and markers of LV filling pressure (E to lateral Ea ratio, E:Vp, and BNP). Conversely, arterial compliance was directly related to body size and echocardiographic parameters of systolic and diastolic function. Reduced arterial compliance was also associated with female sex but was not related to body mass index or LV mass indexed to either height or body surface area.

Multivariate analysis confirmed arterial compliance to be independently related to body surface area, pulse pressure, and $\mathrm{Vp}$ in this population (table 3 ). The class of antihypertensive agent used was not related to arterial compliance.

\section{Relations of arterial compliance in patients with diastolic dysfunction}

In the subgroup of patients with diastolic dysfunction, arterial compliance was most strongly related to pulse

Table 4 Multivariate predictors of arterial compliance in the subgroup of patients with diastolic dysfunction

\begin{tabular}{lll}
\hline Predictor & $\boldsymbol{\beta}$ & $\mathbf{p}$ Value \\
\hline Age & -0.206 & 0.032 \\
Height & 0.173 & 0.066 \\
Pulse pressure & -0.614 & $<0.001$ \\
Lateral Ea & 0.219 & 0.031 \\
Vp & 0.210 & 0.033 \\
\hline$R^{2}=0.77$ & & \\
\hline
\end{tabular}

Table 5 Multivariate predictors of lateral Ea velocity in the subgroup of patients with diastolic dysfunction

\begin{tabular}{lll}
\hline Predictor & $\boldsymbol{\beta}$ & $\mathbf{p ~ V a l u e}$ \\
\hline Sex & 0.283 & 0.034 \\
Diastolic blood pressure & -0.520 & $<0.001$ \\
Arterial compliance & 0.303 & 0.025 \\
\hline$R^{2}=0.52$. & & \\
\hline
\end{tabular}

pressure (fig 2). Arterial compliance was moderately correlated with both the lateral Ea (fig 2) and the septal systolic mitral annular velocity $(r=0.42, \mathrm{p}=0.011)$. Arterial compliance was also directly related to sex $(r=0.38$, $\mathrm{p}=0.020)$, height $(r=0.41, \mathrm{p}=0.012)$, and $\mathrm{Vp}$ $(r=0.27, \mathrm{p}=0.100)$ and inversely related to age $(r=-0.39, \quad \mathrm{p}=0.017)$, systolic blood pressure $(r=-0.69, \mathrm{p}<0.001)$, and the ratio of $\mathrm{E}$ to lateral Ea $(r=-0.44, \mathrm{p}=0.006)$. A multivariate model of these variables (excluding co-linear variables) showed arterial compliance to be independently related to age, pulse pressure, and diastolic function (both lateral Ea and Vp) (table 4).

With lateral Ea used as a marker of global diastolic function, the univariate correlates of diastolic dysfunction were sex, body mass index, systolic and diastolic blood pressures, and arterial compliance. In multivariate analysis of these variables, the independent predictors of diastolic dysfunction were sex, diastolic blood pressure, and arterial compliance (table 5). Each of these parameters remained significantly related to Ea when body surface area was added to the model, which increased $R^{2}$ to 0.57 . Arterial compliance remained an independent predictor of diastolic dysfunction ( $\beta=0.458, p=0.001)$ when age and LV mass were included in the multivariate analysis. A second multivariate model examining Vp as a marker of global diastolic function gave similar results. In this model, Vp was independently associated with systolic blood pressure $(p=0.038)$ and arterial compliance $(\mathrm{p}=0.009)$.

\section{Relation of sex to arterial compliance and diastolic function}

Table 6 details sex differences in clinical and echocardiographic variables for the 70 hypertensive patients. Despite being well matched for age, body mass index, and blood pressure, women had reductions in several parameters of diastolic function. Arterial compliance was significantly lower in women, including when indexed to height. Similar findings were present in the subgroup of patients with diastolic dysfunction. In this group, sex correlated significantly with both lateral Ea $(r=0.33, \mathrm{p}=0.046)$ and arterial compliance $(r=0.38, \mathrm{p}=0.020)$. In the multivariate model described above (table 5 ), which did not examine interactions, sex was independently related to lateral Ea after adjustment for other variables. In a separate model, the combination of sex and arterial compliance in an interaction term was highly significant $(p=0.001)$ and resulted in slightly greater predictive value for Ea $\left(R^{2}=0.54\right)$.

\section{DISCUSSION}

The study shows that in a relatively homogeneous and ambulatory hypertensive population, progressively abnormal diastolic function is associated with lower arterial compliance. In patients with established diastolic dysfunction, arterial compliance is inversely related to age and blood pressure and directly related to LV diastolic function. However, diastolic function is known to be influenced by 


\begin{tabular}{|c|c|c|c|}
\hline Variable & Men & Women & p Value \\
\hline Age (years) & $57(8)$ & $59(8)$ & 0.20 \\
\hline Body mass index $\left(\mathrm{kg} / \mathrm{m}^{2}\right)$ & $29.9(4.7)$ & $30.3(5.9)$ & 0.75 \\
\hline Systolic blood pressure $(\mathrm{mm} \mathrm{Hg})$ & 146 (19) & $151(22)$ & 0.31 \\
\hline Diastolic blood pressure (mm Hg) & $82(8)$ & $81(12)$ & 0.58 \\
\hline Pulse pressure $(\mathrm{mm} \mathrm{Hg})$ & 63 (15) & $70(18)$ & 0.11 \\
\hline LV mass/body surface area $\left(\mathrm{g} / \mathrm{m}^{2}\right)$ & $86(20)$ & 79 (15) & 0.12 \\
\hline LV mass $/$ height ${ }^{2.7}\left(\mathrm{~g} / \mathrm{m}^{2.7}\right)$ & $39.3(10.8)$ & $39.6(9.7)$ & 0.88 \\
\hline Relative wall thickness & $0.40(0.05)$ & $0.38(0.06)$ & 0.16 \\
\hline Left atrial area/height $\left(\mathrm{cm}^{2} / \mathrm{m}^{2}\right)$ & $13.8(2.5)$ & $13.1(2.4)$ & 0.24 \\
\hline$E: A$ & $1.12(0.36)$ & $0.97(0.27)$ & 0.059 \\
\hline Pulmonary vein $A$ reversal $(\mathrm{cm} / \mathrm{s})$ & $29.1(3.9)$ & $31.8(5.3)$ & 0.021 \\
\hline Septal Sa $(\mathrm{cm} / \mathrm{s})$ & $6.4(1.5)$ & $6.3(1.3)$ & 0.83 \\
\hline Lateral Ea $(\mathrm{cm} / \mathrm{s})$ & $8.8(1.8)$ & $8.0(1.6)$ & 0.045 \\
\hline$V_{p}(\mathrm{~cm} / \mathrm{s})$ & $64(26)$ & $57(22)$ & 0.23 \\
\hline E:lateral Ea & $8.0(2.1)$ & $8.8(2.5)$ & 0.12 \\
\hline Arterial compliance $(\mathrm{ml} / \mathrm{mm} \mathrm{Hg})$ & $1.10(0.40)$ & $0.83(0.37)$ & 0.005 \\
\hline Arterial compliance/height $\left(\mathrm{ml} / \mathrm{mm} \mathrm{Hg} / \mathrm{m}^{2}\right)$ & $0.63(0.23)$ & $0.52(0.23)$ & 0.042 \\
\hline
\end{tabular}

several interacting factors including age, sex, blood pressure, and LV hypertrophy, ${ }^{17}$ all of which may potentially influence arterial compliance. In a multivariate model adjusting for these other variables, the study showed that arterial compliance was an independent predictor of LV diastolic dysfunction (as assessed by both Ea and Vp). In fact, arterial compliance and blood pressure were the only variables that were independently associated with diastolic dysfunction, consistent with a possible mechanistic link between arterial compliance and diastolic HF.

Several plausible pathways exist whereby arterial compliance may contribute to the pathological changes in the LV that form the substrate for diastolic dysfunction (fig 3). Increased stiffness of conduit arteries is associated with higher velocity of transmission of the pulse wave generated by LV ejection; early return of reflected waves that arrive back at the heart during LV systole may lead to augmentation of the central aortic pressure wave amplitude, thus increasing LV afterload and central pulse pressure. ${ }^{1}$ Increased afterload may promote myocyte hypertrophy and may also directly slow LV relaxation. ${ }^{18}$ The concomitant reduction in central diastolic blood pressure may compromise coronary perfusion, which, in association with myocyte hypertrophy and increased afterload, may exacerbate subendocardial ischaemia. This can further impair myocardial relaxation and promote interstitial fibrosis leading to reduced LV compliance. Indeed, increased ventricular stiffness in association with arterial stiffening has recently been shown in patients with overt diastolic HF, and interaction of these processes may be important in the clinical expression of HF. ${ }^{19}$ Furthermore, in patients with presumed diastolic HF (clinical HF with preserved ejection fraction), Hundley et al ${ }^{2}$ observed reduced proximal aortic distensibility, which correlated strongly with exercise intolerance.

Similar findings to those of the present study have recently been reported in a more heterogeneous group. ${ }^{20}$ In a population including normal subjects and patients with various conditions, Vinereanu et $a l^{20}$ showed that arterial stiffness was inversely related to long axis LV function (systolic and early diastolic mitral annular velocities) and Vp. These authors also showed that arterial stiffness was independently related to Ea and suggested a possible causal link through the promotion of subendocardial ischaemia. ${ }^{20}$ Previous studies in hypertensive patients have also reported an association between arterial stiffness and LV structural changes, including concentric remodelling and hypertrophy, ${ }^{21}{ }^{22}$ which are themselves associated with diastolic dysfunction..$^{23} 24$
The relation between arterial compliance and diastolic dysfunction may be particularly important with respect to hypertensive women, who have a higher prevalence of diastolic HF than do men. ${ }^{25-27}$ Experimental and clinical studies suggest that in response to increased afterload, women exhibit a greater degree of concentric remodelling, ${ }^{28} 29$ which as discussed is associated with both arterial stiffness and diastolic dysfunction. In addition, several studies have reported increased values of arterial stiffness in women. ${ }^{3031}$ In the present study sex was associated with reduced Ea velocity and independently related to arterial compliance in hypertensive patients (table 3). After adjustment for other factors, female sex was independently predictive of diastolic dysfunction. In addition, the results indicate that an interaction between sex and arterial compliance may be important, such that arterial dysfunction may contribute to diastolic dysfunction in hypertensive women to a greater extent than in hypertensive men.

Aortic stiffness is an independent predictor of all cause and cardiovascular mortality in patients with essential hypertension. ${ }^{32}$ Although the mechanisms of this increased risk are not fully understood, the promotion of diastolic HF (itself associated with increased risk) ${ }^{26}$ is one potential mechanism. Although a causal relation was not investigated in this study,

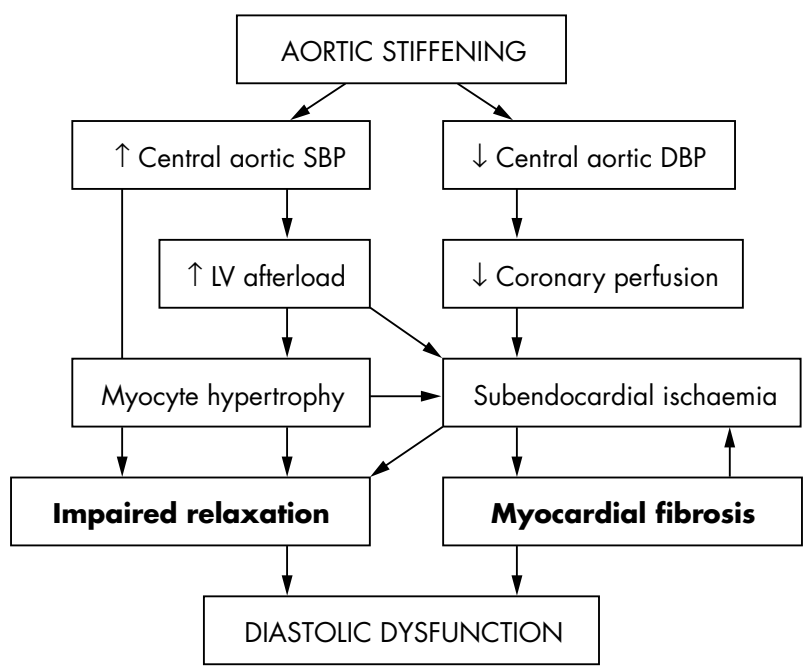

Figure 3 Pathophysiological pathways through which aortic stiffness may contribute to the development of diastolic dysfunction. DBP, diastolic blood pressure; SBP, systolic blood pressure. 
the independent association of increased arterial compliance with linear parameters of diastolic function supports a mechanistic link between these two pathophysiological entities in hypertensive patients with exertional dyspnoea who have diastolic dysfunction.

\section{Conclusion}

In hypertensive patients with exertional dyspnoea, arterial compliance is independently related to the degree of diastolic dysfunction and may be particularly important to the development of diastolic HF in women. Therefore, as arterial compliance (like LV diastolic dysfunction) may be modifiable, it should be considered a potential target for intervention in future studies of diastolic HF.

\section{Authors' affiliations}

P M Mottram, B A Haluska, R Leano, S Carlier, C Case, T H Marwick, University of Queensland, Brisbane, Queensland, Australia

Supported in part by a scholarship and grant in aid from the National Heart Foundation of Australia

\section{REFERENCES}

1 O'Rourke MF. Diastolic heart failure, diastolic left ventricular dysfunction and exercise intolerance. J Am Coll Cardiol 2001;38:803-5.

2 Hundley WG, Kitzman DW, Morgan TM, et al. Cardiac cycle-dependent changes in aortic area and distensibility are reduced in older patients with isolated diastolic heart failure and correlate with exercise intolerance. J Am Coll Cardiol 2001;38:796-802.

3 Lubien E, DeMaria A, Krishnaswamy P, et al. Utility of B-natriuretic peptide in detecting diastolic dysfunction: comparison with Doppler velocity recordings Circulation 2002; 105:595-601

4 Devereux RB, Alonso DR, Lutas EM, et al. Echocardiographic assessment of left ventricular hypertrophy: comparison to necropsy findings. Am J Cardiol 1986;57:450-8.

5 Rakowski H, Appleton C, Chan KL, et al. Canadian consensus recommendations for the measurement and reporting of diastolic dysfunction by echocardiography: from the Investigators of Consensus on Diastolic Dysfunction by Echocardiography. J Am Soc Echocardiogr 1996;9:736-60.

6 Nishimura RA, Tajik AJ. Evaluation of diastolic filling of left ventricle in health and disease: Doppler echocardiography is the clinician's Rosetta stone. J Am Coll Cardiol 1997;30:8-18.

7 De Marchi SF, Bodenmuller M, Lai DL, et al. Pulmonary venous flow velocity patterns in 404 individuals without cardiovascular disease. Heart 2001;85:23-9.

8 Kuecherer HF, Muhiudeen IA, Kusumoto FM, et al. Estimation of mean left atrial pressure from transesophageal pulsed Doppler echocardiography of pulmonary venous flow. Circulation 1990;82:1127-39.

9 Sohn DW, Chai IH, Lee DJ, et al. Assessment of mitral annulus velocity by Doppler tissue imaging in the evaluation of left ventricular diastolic function. J Am Coll Cardiol 1997;30:474-80.

10 Garcia MJ, Smedira NG, Greenberg NL, et al. Color M-mode Doppler flow propagation velocity is a preload insensitive index of left ventricular relaxation: animal and human validation. J Am Coll Cardiol 2000;35:201-8.

11 Ommen SR, Nishimura RA, Appleton CP, et al. Clinical utility of Doppler echocardiography and tissue Doppler imaging in the estimation of left ventricular filling pressures: a comparative simultaneous Dopplercatheterization study. Circulation 2000;102:1788-94.

12 Nagueh SF, Middleton KJ, Kopelen HA, et al. Doppler tissue imaging: a noninvasive technique for evaluation of left ventricular relaxation and estimation of filling pressures. J Am Coll Cardiol 1997;30:1527-33.

13 Garcia MJ, Thomas JD, Klein AL. New Doppler echocardiographic applications for the study of diastolic function. J Am Coll Cardiol 1998;32:865-75

14 Stergiopulos N, Meister JJ, Westerhof N. Simple and accurate way for estimating total and segmental arterial compliance: the pulse pressure method. Ann Biomed Eng 1994;22:392-7.

15 Chen $\mathrm{CH}$, Nevo $\mathrm{E}$, Fetics $\mathrm{B}$, et al. Estimation of central aortic pressure waveform by mathematical transformation of radial tonometry pressure: validation of generalized transfer function. Circulation 1997;95:1827-36.

16 Segers $\mathbf{P}$, Carlier S, Pasquet A, et al. Individualizing the aorto-radial pressure transfer function: feasibility of a model-based approach. Am J Physiol Heart Circ Physiol 2000;279:H542-9.

17 Kitzman DW. Why is diastolic heart failure in older patients the cardiologist's enigma? Dialogues Cardiovasc Med 2001;6:95-103.

18 Leite-Moreira AF, Correia-Pinto J, Gillebert TC. Afterload induced changes in myocardial relaxation: a mechanism for diastolic dysfunction. Cardiovasc Res 1999:43:344-53.

19 Kawaguchi M, Hay I, Fetics B, et al. Combined ventricular systolic and arterial stiffening in patients with heart failure and preserved ejection fraction: implications for systolic and diastolic reserve limitations. Circulation 2003;107:714-20.

20 Vinereanu D, Nicolaides E, Boden L, et al. Conduit arterial stiffness is associated with impaired left ventricular subendocardial function. Heart 2003;89:449-50.

21 Roman MJ, Ganau A, Saba PS, et al. Impact of arterial stiffening on left ventricular structure. Hypertension 2000;36:489-94.

22 Palmieri V, Bella JN, Roman MJ, et al. Pulse pressure/stroke index and left ventricular geometry and function: the LIFE Study. J Hypertens 2003;21:781-7.

23 Ren JF, Pancholy SB, Iskandrian AS, et al. Doppler echocardiographic evaluation of the spectrum of left ventricular diastolic dysfunction in essential hypertension. Am Heart J 1994;127:906-13.

24 Qu P, Ding Y, Xia D, et al. Variations in cardiac diastolic function in hypertensive patients with different left ventricular geometric patterns. Hypertens Res $2001 ; 24: 601-4$

25 Kitzman DW, Gardin JM, Gottdiener JS, et al. Importance of heart failure with preserved systolic function in patients $>$ or $=65$ years of age. CHS Research Group. Cardiovascular health study. Am J Cardiol 2001;87:413-9.

26 Vasan RS, Larson MG, Benjamin EJ, et al. Congestive heart failure in subjects with normal versus reduced left ventricular ejection fraction: prevalence and mortality in a population-based cohort. J Am Coll Cardiol 1999:33:1948-55.

27 Masoudi FA, Havranek EP, Smith G, et al. Gender, age, and heart failure with preserved left ventricular systolic function. J Am Coll Cardiol 2003:41:217-23.

28 Krumholz HM, Larson M, Levy D. Sex differences in cardiac adaptation to isolated systolic hypertension. Am J Cardiol 1993;72:310-3.

29 Aurigemma GP, Gaasch WH. Gender differences in older patients with pressure-overload hypertrophy of the left ventricle. Cardiology 1995;86:310-7.

30 Gatzka CD, Kingwell BA, Cameron JD, et al. Gender differences in the timing of arterial wave reflection beyond differences in body height. J Hypertens $2001 ; 19: 2197-203$

31 Waddell TK, Dart AM, Gatzka CD, et al. Women exhibit a greater agerelated increase in proximal aortic stiffness than men. $J$ Hypertens $2001 ; 19: 2205-12$

32 Laurent S, Boutouyrie P, Asmar R, et al. Aortic stiffness is an independent predictor of all-cause and cardiovascular mortality in hypertensive patients. Hypertension $2001 ; 37: 1236-41$. 